\title{
5
}

\section{METEOROID POPULATIONS AND ORBITS}

\author{
Z. CEPLECHA
}

Statistical studies of the photographic data on atmospheric trajectories and orbits of meteors (from $10^{-4} \mathrm{~g}$ to hundreds of tons) point to 5 groups of bodies with different structure and composition. The following classification is proposed: Fireball group I E exceptional cases of so-called "asteroidal" bodies among Super-Schmidt (SS) and small-camera (SC) meteors Đordinary chondrites. Fireball group $I I \equiv$ group $A$ among $S S$ and $S C=$ carbonaceous chondrites. Group $B$ among $S S$ and $S C \equiv$ dense cometary materials with small perihelion distances (not distinguishable among fireballs). Fireball group IIIa $\equiv$ group $C$ among $S S$ and $S C \equiv$ regular cometary material. Fireball group IIIB $\equiv$ group $D$ (above C) of SS and SC $\equiv$ Draconid-shower type of cometary material. Statistics of the orbits for separate groups is considered and characteristic orbits are given.

I want to speak about different populations of meteoroids in the mass range from $10^{-4}$ to $10^{8} \mathrm{~g}$ and about their relations to the other bodies of the solar system. All we know about them have to be derived from their atmospheric trajectories. Only during this extremely short interval of their lifetime, these meteoroids are accessible for observations. The collision of the meteoroid with the atmosphere gives rise to a luminous trajectory of a duration of a few tenth to some ten seconds for these masses. The meteoroid penetrating into the atmosphere with initial velocity somewhere from 72 to $11 \mathrm{~km} / \mathrm{s}$ is first heated up to the point, where a severe ablation and evaporation begins. This heating goes up exponentially approximately following the uprise of the air density. After the ablation starts, the gas close to the body is mainly composed of hot meteoroid vapors emitting the light, which can be observed on the Earth's surface. Thus we do not actually observe the meteoroid itself, but some luminous volume surrounding and following it.

We usually denote the height, where we begin to observe the light of a meteor, as its beginning height. After all the mass is ablated or the velocity slowed down to few kilometers per second, the meteor phenomenon is terminated and we denote the corresponding height as the terminal or the end height.

If we would have two meteoroids of the same initial velocity, mass and the same angle of incidence, then any observed difference in the beginning height and the terminal height should be associated with the difference in the meteoroid structure and composition. Even if the situation is complicated by different initial velocity, mass and angle of incidence, still the study of meteor beginning and terminal heights is a good and rather simple mean for distinguishing among meteoroid populations of different composition and structure. At the same time the initial velocity as a vector (the direction opposite to the 
velocity vector is usually denoted as meteor radiant by meteor astronomers) and the known position of the Earth in its orbit give us all we need to compute the meteoroid orbit. We can then compare statistics of orbits with statistics of beginning and terminal heights, which results in orbital characteristics and orbital differences among meteoroid populations (groups).

To do so, we need good and precise observations of the meteor phenomenon. Moreover we need homogeneous material for statistical purposes. The photographic data obtained from at least two stations separated by several tens of kilometers (or even few hundreds of kilometers for fireballs) are the best possibility. Such observations give us data on heights of individual points of the meteor trajectory, velocities, decelerations and light intensities. The meteoroid mass is determined from the integrated light intensity. In principle three different types of cameras used for recording meteors resulted in three statistical sets of data. These sets have already been used separately to study meteoroid populations. This review is an attempt to intercompare all the results and to propose a classification of meteoroid populations valid for the entire mass-range.

The oldest results were obtained using classical small-cameras. The Harvard double-station program initiated by Fred L. Whipple in 1936 (Whipple 1938, 1954; Jacchia et al. 1965) gave the first results on small-camera meteors usable for statistical purposes. In this review I complemented this observational material of the small-camera meteors by observations from Dushanbe, Odessa and Ondrejov; altogether 364 sporadic meteors were used. (See Ceplecha 1965, p. 47 to 51 for details). The small-camera meteors have typical masses from $10^{-1}$ to $10^{3} \mathrm{~g}$.

Thanks to the endeavor of F. L. Whipple, photographic data on smaller meteoroids became available, after the project of Baker Super-Schmidt cameras had been accomplished by the Harvard Observatory (Jacchia and Whipple 1956). The precisely reduced orbits of 413 faint photographic meteors with longer trajectories proved to be highly selective in favor of the classical cometary population. Thus I use here the more complete material of 2529 meteors photographed by Super-Schmidt cameras and reduced and published by McCrosky and Posen (1961). (See Ceplecha (1967) page 35 to 47 for details). This material proved to be homogeneous and very good for statistical purposes. The SuperSchmidt meteors have a typical mass-range from $10^{-3}$ to $10 \mathrm{~g}$.

The third observational material available only recently is that on fireballs. Fred L. Whipple was again with it as the director of the Smithsonian Astrophysical Observatory, when the effort of R. E. McCrosky resulted in operating the Prairie Network for fireballs. (McCrosky and Posen 1968). A recent study of populations among fireballs were based on data of 232 events photographed by the Prairie Network during 7 years. (Ceplecha and McCrosky 1976). The typical mass-range for fireballs is from $10^{2}$ to $10^{6} \mathrm{~g}$.

Different meteoroid populations were first recognized independently by Jacchia (1958) and Ceplecha (1958). The differences in beginning heights proved to be the most important tool for recognition of different meteoroid populations among Super-Schmidt and small-camera meteors, when and if the right dependence on velocity was considered. (Ceplecha 1967, 1968; Cook 1973). Two main discrete levels of meteor beginning heights separated by $10 \mathrm{~km}$ difference has been found. The lower level was denoted A, the higher was denoted $C$. The C-group of meteoroids was recognized to contain two populations of orbits: one with ecliptically concentrated short-period orbits was denoted $C_{1}$ and the other with random orbital inclinations of long-period orbits was denoted $\mathrm{C}_{2}$. The classical meteor showers with known parent comets are of the type $\mathrm{C}_{1}$ and $\mathrm{C}_{2}$; thus the cometary origin of meteoroids of the whole C-group is quite certain.

The question of meteoroid bulk densities for separate groups proved to be more complicated. The A-group was recognized to contain meteoroids of about 3 times greater bulk densities than the $\mathrm{C}$-group. The problem with calibration 
METEOROIDS

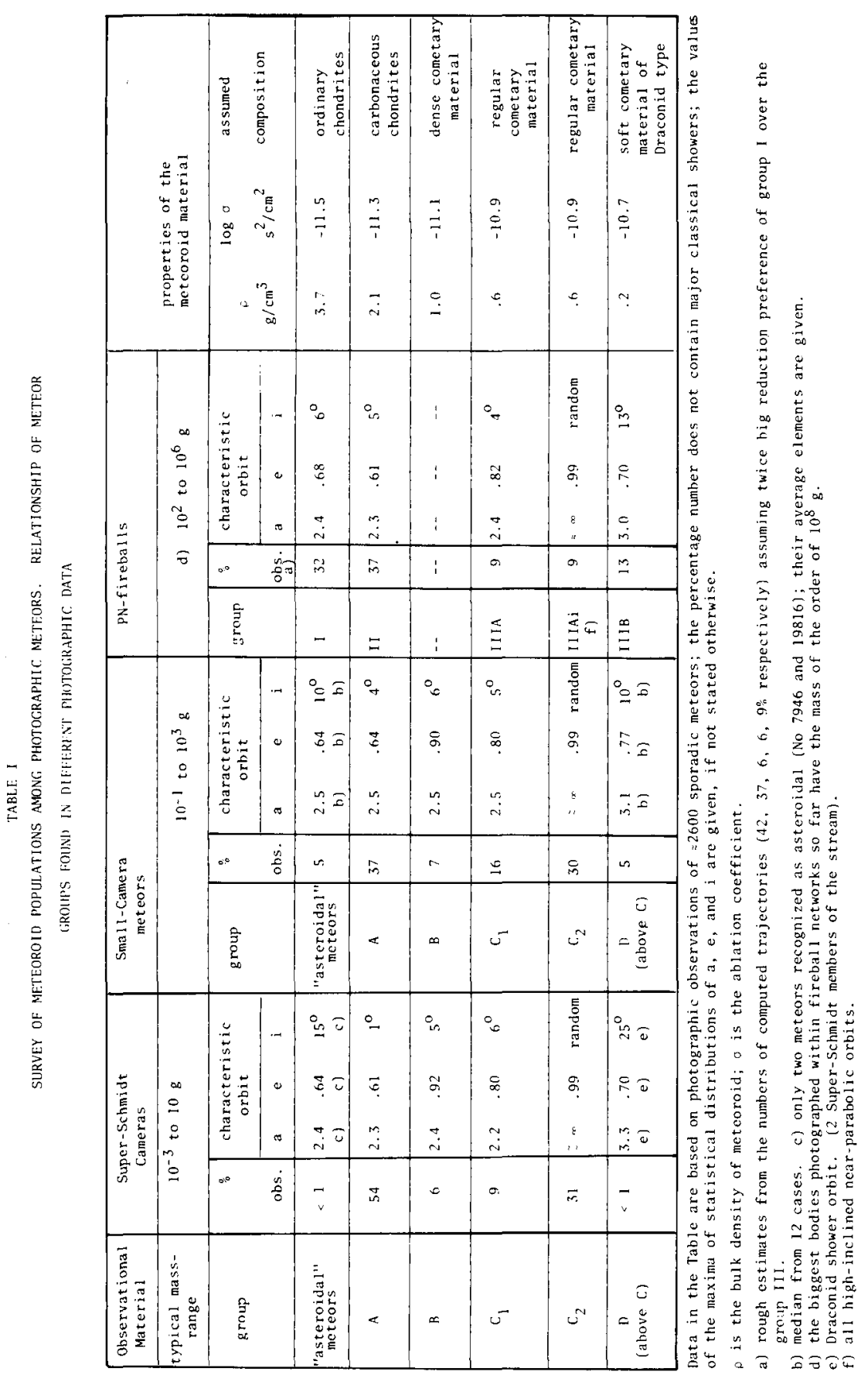


of these relative values by bodies of "known" composition was the reason of overestimated densities by Ceplecha $(1967,1968)$. The problem of the velocity dependence of meteor beginning heights and calibration problems was the reason of underestimated densities by Verniani (1965, 1967) and Jacchia et al. (1965). The calibration through the data on fireball populations with knowing the actual bodies in case of the Pribram and the Lost City meteorites, gave the values of densities intermediate to both the extreme results. The A-group contains bodies with average bulk densities of about $2.1 \mathrm{~g} / \mathrm{cm}^{3}$, while the $\mathrm{C}$-group contains bodies with average densities of $0.6 \mathrm{~g} / \mathrm{cm}^{3}$ (Table I).

Two additional smaller groups were found among Super-Schmidt and smal1camera meteors. The intermediate group $B$ has typical orbits with small perihelion distances and aphelion close to Jupiter. There seems to be not a direct cometary association to meteoroids of this group, but the Geminid shower is a typical member of the B-group, and as far as Geminids are assumed to be cometary in origin, the whole group $B$ should be associated with comets. According to Cook (1973) the B-group meteoroids should be associated with less dense cores of smaller cometary nuclei that have lost their surfaces and are too small to have been observed. The bulk density of meteoroids of this group should be close to $1 \mathrm{~g} / \mathrm{cm}^{3}$.

The other smaller group was referred to as a group "above $C$ " accounting for very high beginning heights. The Draconid meteor shower is a typical member of this group. I will denote this group as D-group. It contains extremely soft meteoroids with the lowest known densities from all types of solid cometary material coming to the Earth $\left(0.2 \mathrm{~g} / \mathrm{cm}^{3}\right)$.

Very few meteoroids among Super-Schmidt and small-camera meteors penetrated deep into the atmosphere and indicated thus stronger structure and density than those of the A-group. These meteors were called by some authors "asteroidal," which is not a good name presuming the origin. The average bulk density of these bodies is $3.7 \mathrm{~g} / \mathrm{cm}^{3}$ in the density scale used in this review (Table I).

Except bulk density, another parameter characterizing the composition and structure of the meteor body is the ablation coefficient $\sigma=\Lambda /(2 \Gamma \xi)$, where $\Lambda$ is the heat-transfer coefficient, $\Gamma$ the drag coefficient and $\xi$ the energy necessary for ablation of $1 \mathrm{~g}$ of the meteoroid material. The ablation coefficient is a quantitative measure how readily the meteoroid ablates. Knowing the mass and velocity, the ablation coefficient can be determined from the photographic observations of meteors. The A-group of meteoroids contains bodies with ablation coefficient 3 times smaller than the C-group. Thus the meteoroids of the C-group are more readily ablated and even a big initial mass cannot penetrate deep into the atmosphere.

The attempt to study the firebarls the same way as the fainter meteors using the beginning heights proved to be useless. But different penetrating abilities were found to be a good criterion for distinguishing among different fireball populations. Thus the end heights turned out to be the most important for recognition of fireball populations. The main difference of this approach is an additional mass dependence of the used criteria. Also the problem of significant terminal mass was encountered, which had been negligible for fainter meteors.

Four groups of different composition and structure were found among fireballs (Ceplecha and McCrosky 1976) and were denoted by Roman numerals eventually with a letter suffix. Group $I$ is of the greatest density and contains Príbram (Ceplecha 1961) and Lost City (McCrosky et al. 1971) meteorites, the only two meteorite falls photographed. Thus the average bulk density of fireballs of group I was assumed $3.7 \mathrm{~g} / \mathrm{cm}^{3}$ using data of these two meteorites as safe calibration values. Group I is evidently connected with ordinary chondrites.

The meteoroids of the group II have a somewhat lower density of $2.1 \mathrm{~g} / \mathrm{cm}^{3}$ 
and they are very likely belonging to carbonaceous chondrites. Except for the reasons given in favor of this identification in the paper by Ceplecha and McCrosky (1976), there is a brand-new evidence from spectral records of the Kamyk fireball obtained at the Ondrejov Observatory in March 1976. (This fireball was photographed from 11 stations of the European Network for fireballs; Ceplecha et al. 1977). This fireball penetrated to $32 \mathrm{~km}$ and was a sure member of the group II; the extremely good spectral $(15 \AA / \mathrm{mm})$ contain more than 500 features in the visible region and show relatively strong emissions of the $\mathrm{CN}$ molerile demonstrating the abundance of carbon.

Firebalis of the groups IIIA and IIIAi have densities of $0.6 \mathrm{~g} / \mathrm{cm}^{3}$ and have direct evidence of cometary origin through showers. The group IIIA contains orbits of short period and ecliptic concentration and the group IIIAi contains long-period randomly-inclined orbits. A very good spectrum of a fireball of the group IIIAi was published (Ceplecha 1971) and relatively strong emissions of $\mathrm{CN}$ were found. Thus also low density cometary material in longperiod orbits does contain carbon.

The fireballs of the group IIIB have densities of $0.2 \mathrm{~g} / \mathrm{cm}^{3}$ and they contain fireballs of the Draconid meteor shower. This is a very distinct group and contains relatively quite a number of fireballs.

The interrelations of all the groups is presented in Table I. Altogether 6 populations are present with 5 different compositions among the meteoroids in the considered mass-range. The average bulk density and ablation coefficients are given for each group and a probable composition and structure is proposed.

The identification of the fireball group I and the so-called "asteroidal" meteors with the ordinary chondrites is mainly based on the two photographed meteorite fa11s: Pribram and Lost City. The identification of the group IIIB and of the group D with the Draconid shower is based on observed members of this shower in all three observational materials. The C-group meteors and the IIIA group meteors are easily identified as regular cometary material through showers with known parent comets. The identification of the A group with the group II and with the carbonaceous chondrites comes out then quite naturally and moreover it is supported by the spectral evidence already mentioned.

Table I contains "characteristic" orbits for each of the groups. To avoid any misunderstanding I strongly stress that these values are mostly maxima of broad irregular statistical distributions (sometimes medians as marked in Table I). It is not possible to separate all these populations using only the orbital elements.

There is not much difference in semimajor axes for ordinary chondrites, carbonaceous chondrites and cometary material with ecliptic concentrations; all values are from 2.2 to $2.5 \mathrm{~A} . \mathrm{U}$. The Draconid-shower type of material has longer semimajor axes of slightly more than $3 \mathrm{~A} . \mathrm{U}$. The cometary material with randomly inclined orbits has of course very large semimajor axes, which is symbolicly denoted by $\simeq \infty$ in Table $I$.

The orbital distinction among groups is mostly in eccentricities: ordinary and carbonaceous chondrites have the orbits with the smallest eccentricity, 0.6 . The Draconid type of material has somewhat higher eccentricities, 0.7 , the regular cometary material with ecliptic concentration even higher, 0.8 , followed by the dense cometary material of the B-group, 0.9 , and by the cometary material with randomly inclined orbits with eccentricity approaching 1.

There is a very little difference in characteristic orbits of ordinary chondrites and of carbonaceous chondrites. But the statistical distribution of the semimajor axes is strikingly different (Fig. 1). The number of observed orbits of ordinary chondrites increases almost linearly with their semimajor axes starting with $a=1 \mathrm{~A} . \mathrm{U}$. and then suddenly drops forming a cut-off at 2.7 A.U. The decrease is 7 times steeper than the increase! The carbonaceous chondrites have a symmetrical distribution with maximum at $2.3 \mathrm{~A} . \mathrm{U}$. and still 
enough orbits with $a=3$ A.U. A smal1 second maximum in the distribution of semimajor axes for carbonaceous chondrites near $1.2 \mathrm{~A}$.U. (quite missing for the regular chondrites) may indicate a secondary Earth's or Moon's component.

The Pribram meteorite has the orbit very close to the maxima of distribution for ordinary chondrites, while the Lost City meteorite is on ascending parts of the distributions of a and e. Especially eccentricity of the Lost City orbit is much lower than is the average. The orbit of Pribram is more typical for the ordinary chondrites than that of the Lost City.

Because the so-called "asteroida1" meteors are very rare among faint meteors, the distributions of the orbital elements of the group-I fireballs are actually the first hint at statistics of the orbits of ordinary chondrites from photographic records. The fireball-group-I data can be considered as representative of ordinary chondrites. The orbital data for any detailed study should be taken from the original paper (Ceplecha and McCrosky 1976).

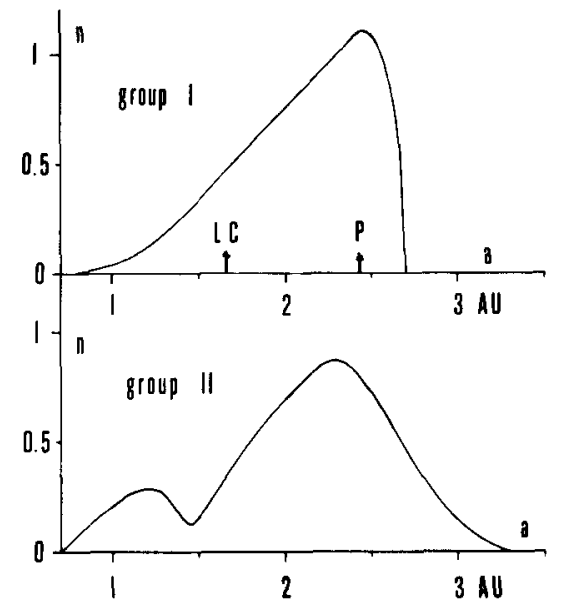

Figure 1. Distribution of semimajor axes, a, of group I fireballs lordinary chondrites) and group II fireballs (carbonaceous chondrites). The scale of the relative number of firebalis, $n$, is chosen so that the integral number of all cases is equal 1 . LC is the Lost City meteorite, $P$ is the Pribram meteorite.

The inclinations for all groups except $C_{2}$ and IIIAi show an ecliptical concentration, but the maxima of distributions are not at $0^{\circ}$ exactly. They are usually between $0^{\circ}$ and $10^{\circ}$. This may be due to planetary collisions and perturbations as already pointed out by McCrosky and Posen (1961). The only higher inclination than $10^{\circ}$ at the maximum of distribution is that of the Draconid type group.

The parent bodies of the softest Draconid type material are short period comets, one of them being the Giacobini-Zinner belonging to the Draconid meteor shower. Meteoroids of this type are very rare among the observed faint bodies and are growing in number toward the big masses forming $13 \%$ of the observed fireballs.

The parent bodies of the $\mathrm{C}_{1}$ and IIIA group are short-period comets again; most of the classical meteor showers are of this type and a lot of direct wellestablished cometary associations is available. This group forms $9 \%$ of all Super-Schmidt meteors; it increases to maximum of $16 \%$ from the smal1-camera meteors and again becomes less significant with $9 \%$ from the fireballs. Also 
the classical meteor showers are the most distinct and numerous among the sma11-camera meteors.

The parent bodies of the $\mathrm{C}_{2}$ and IIIAi group are long-period comets; showers as e.g. Perseids with association to comet Swift-Tuttle 1862 III are within this group and one can hardly doubt the cometary origin of it. The Super-Schmidt and small-camera meteors contain $30 \%$ of this long-period cometary component, which decreases to $9 \%$ for fireballs.

The parent bodies of the B-group have to be of the same nature as the parent body of the Geminid meteor shower. The cometary origin is highly probable. The B-group forms 6 and 7\% of all faint meteors and was not separated among fireba1ls.

The A-group and the group II belonging to carbonaceous chondrites are the most numerous among all the studied masses. They form $54 \%$ of all observed Super-Schmidt meteors, $37 \%$ of the smal1-camera meteors and $32 \%$ of the fireballs. Cook (1973) prefers the cometary origin of this group ascribing it to the core of a cometary nucleus. A strong support to this view is the one existing association of a meteor shower of type A with a known comet: $\tau$ Herculids (Lindblad 1971) with the comet Schwassman-Wachmann 3 . Its eventual observation in 1979 return may cast some more light on the problem. The scarcity of ordinary chondrites among smaller meteoroids in contrast to the abundance of carbonaceous chondrites points to different origin. The differences in the distribution of semimajor axes (Fig. 1) support this view. The cometary origin for carbonaceous chondrites seems to be a strong possibility. If carbonaceous chondrites are not cometary, they should at least be connected with different type of objects than the ordinary chondrites.

The parent bodies of the ordinary chondrites are mostly believed to be asteroidal bodies (Anders 1971). The difficulties with this view from the point of Earth crossing orbits are discussed in many papers of this Colloquium.

The importance of the carbonaceous chondrites among all sizes of the studied meteoroids is evident. This is well demonstrated by the ratio of cometary to asteroidal meteoroids. If we assume that carbonaceous chondrites are of cometary origin, then the observed ratio of the bodies with cometary to the bodies with asteroidal origin is $>100$ for meteoroids of $10^{-1} \mathrm{~g}, 20$ for $10 \mathrm{~g}$, and 2 for $10^{4} \mathrm{~g}$. If we assume that the carbonaceous chondrites are of asteroidal origin, the picture is strikingly different. The observed ratio of cometary to asteroidal bodies is then 0.8 for $10^{-1} \mathrm{~g}, 1.4$ for $10 \mathrm{~g}$, and 0.4 for $10^{4} \mathrm{~g}$.

The total mass range, in which the meteoroid populations were studied in this review, is from $10^{-3}$ to $10^{6} \mathrm{~g}$ for almost all the available photographic meteors. Few extreme cases of $10 \mathrm{ow}$ and very big masses outside this mass range that has been photographed can be classified in the same population groups presented here. Adding these extreme wings of the distribution, we can assume the total mass-range of meteoroid studied to be from $10^{-4} \mathrm{~g}$ to hundreds of tons. It is interesting that one of the biggest initial masses on photographic records belongs to the Draconid type (Ceplecha 1976).

At the end I want to mention a paradox in connection with the fireballs and meteorite falls. If somehow we could organize all the incoming meteoroids to be of the same mass, velocity and angle of incidence but having different composition and structure as given in Table $I$, then the meteorite fall of an ordinary chondrite will be preceded by the faintest meteor phenomenon from all the 5 different compositional groups. If we would observe the brightest possible fireball, we may be sure it belongs to the soft Draconid type of material. This material is ablated high in the atmosphere. On the other hand, the faint fireball belonging to the meteorite fall of an ordinary chondrite, would have very long trajectory penetrating very deep into the atmosphere, while the Draconid type would have only very short trajectory ending very high. Thus, an 
observation of a very bright fireball does not necessarily mean a meteorite fal1. The dependence of the brightness on the mass in real situation works partly against the paradox and makes the picture quite complicated. Thus decisions if to search or not to search for meteorites after a bright fireball passage, should be done rather on the basis of criteria, which takes into account the end height, the mass (or brightness) and the velocity with the angle of incidence (Ceplecha and McCrosky 1976).

\section{REFERENCES}

Anders, E. 1971, in Physical Studies of Minor Planets, (ed. T. Gehrels, NASA, Washington), p. 429 .

Babadzanov, P. B., Kramer, E. N. 1963, Ionosphere and Meteors 12, Publ. House Acad. Sci. USSR, Moscow.

Ceplecha, Z. 1958, Bull. Astron. Inst. Czech., 9, 154.

Ceplecha, Z. 1961, Bul1. Astron. Inst. Czech., 12, 21.

Ceplecha, Z. 1967, Smithson. Contr. Astrophys., 11, 35.

Ceplecha, Z. 1968, Smithson. Astrophys. Obs. Spec. Rep. 279.

Ceplecha, Z. 1971, Bull. Astron. Inst. Czech., 22, 219.

Ceplecha, Z. 1976, in, Lecture Notes in Physics 48, (ed. H. Elsässer, H. Fechtig, Springer Verlag, Heidelberg), 385 .

Ceplecha, Z., Bocek, J., Jezkova, M. 1977, Bull. Astron. Inst. Czech., 28, in preparation.

Ceplecha, Z., McCrosky, R. E. 1976, Center for Astrophys., Prepr. Ser. 442.

Cook, A. F. 1973, Smithson. Contr. Astrophys., No. 14.

Jacchia, L. G. 1958, Smithson. Contr. Astrophys., 2, 181 .

Jacchia, L. G., Kopal, Z., and Millman, P. M. 1950, Astrophys. J., 111, 104.

Jacchia, L. G., Verniani, F., and Beiggs, R. E. 1965, Smithson. Astrophys. Obs. Spec. Rep. 175 .

Jacchia, L. G., Whipple, F. L. 1956, in Vistas in Astron. 2, (ed. A. Beer, London), p. 982 .

Jacchia, L. G., Whipple, F. L. 1961, Smithson. Contr. Astrophys., 4, 97.

Kresák, L. 1970, Bu11. Astron. Inst. Czech., 21, 1.

Lindblad, B. A. 1971, Smithson. Contr. Astrophys., No. 12.

Marsden, B. G. 1972, Catalogue of Cometary Orbits, Smithson. Astrophys. Obs., Cambridge.

McCrosky, R. E. 1968, Smithson. Astrophys. Obs. Spec. Rep., 280.

McCrosky, R. E. 1968, in Physics and Dynamics of Meteors, (eds. L. Kresak, P. M. Millman; D. Reidel Pub1. Comp., Dordrecht), p. 265.

McCrosky, R. E. 1971, in Physical Studies of Minor Planets, (ed. T. Gehrels, NASA, Washington), p. 395 .

McCrosky, R. E., Posen, A. 1961, Smithson. Contr. Astrophys., 4, 15.

McCrosky, R. E. 1968, Smithson. Astrophys. Obs. Spec. Rep.. 273.

McCrosky, R. E., Posen, A., Swartz, G., and Shao, C. -Y. 1971, J. Geophys. Res., 76, 4090 .

Vanysek, V. 1971, in Physical studies of Minor Planets, (ed. T. Gehrels, NASA Washington), p. 465 .

Verniani, F. 1965, Smithson. Contr. Astrophys., 8, 141.

Verniani, F. 1967, Smithson. Contr. Astrophys., 10, 181.

Wetheril1, G. W. 1971, in Physical Studies of Minor Planets, (ed. T. Gehrels, NASA, Washington), p. 447 .

Whipple, F. L. 1938, Proc. of the Amer. Philos. Soc., 79, 499.

Whipple, F. L. 1954, Astron. J., 59, 201. 


\section{METEOROIDS}

\section{DISCUSSION}

CHAPMAN: Is it understood what physical process or processes could yield the different size distributions for "asteroidal meteors" and types A/II implied by your table? I would have thought that rapid collisional fragmentation processes would have made the population indices of most small particle populations similar, whatever the sources of the particles.

CEPLECHA: For a long time we know from our observations that faint photographic meteors almost lack the "asteroidal" type of objects which appeared among fireballs as the group I quite well populated. I am an observationalist and I have no real explanation for this.

WHIPPLE: If small chondrites originated with the meteorites (exposure to space) they would have been destroyed by collisions in the typical exposure ages of the meteorites, $\sim 10^{6}$ yr or more. The life times of the interplanetary particles is $\sim 10^{4}$ yr and destruction is mostly by collisions.

CEPLECHA: If one searches for some division between two grossly different origins, then just the absence of the group I bodies in smaller mass-ranges puts the division between the groups $I$ and $I I$.

HUGHES: Verniani found that the density of meteoroids varied as a function of mass $\left(\rho=3.5 \mathrm{~g} \mathrm{~cm}^{-3}\right.$ for $\mathrm{m}<10^{-5} \mathrm{~g} ; \rho=0.8 \mathrm{~g} \mathrm{~cm}$ for $10^{-5}<\mathrm{m}<10^{-1} \mathrm{~g}^{-3} \rho=$ $0.3 \mathrm{~g} \mathrm{~cm}^{-3}$ for $\left.\mathrm{m}>10^{-1} \mathrm{~g}\right)$. How do your results change this conclusion? why was Verniani mistaken?

CEPLECHA: I have no idea about densities of the radar meteors $\left(<10^{-5} g r\right) ; I$ spoke only about the photographic meteors. Verniani's values are only average values, when all well distinguishable groups are dealt with together. This and the problem with calibration are the reasons that Verniani's densities are smaller. I have the opportunity, now, to calibrate the density scale by pírbram and Lost City meteorite densities and to deal with separate groups. To speak about "average" density of all meteoroids in a given mass-range gives not much sense to me. You can compute such a value from the percentage of the groups and from their densities.

MILLMAN: In regard to the mean densities of meteoroids given by Verniani, which are found on the basis of meteor physical theory, I feel that they show the correct relative change as we move from $1 \mathrm{~g}$ objects to those in the $10^{-4}$ to $10^{-6} \mathrm{~g}$ range. However, conclusions of Brownlee and his coworkers, in their study of extraterrestrial micrometeorites, suggest that the absolute densities of Verniani should be increased somewhat. One must also distinguish between whether a density is quoted as a bulk density at a conglomerate or as a density of one of the grains forming this conglomerate.

KRESAK: Your table indicates a conspicuous deficiency of long-period cometary material in the Prairie-Network data. However, these data are biased by a selection of meteors with a minimum duration of about 1 second required for their timing. Would there still remain a considerable disproportion after applying the due correction?

CEPLECHA: I already applied a correction which enhanced 6\% of computed orbits into $9 \%$. If there is some more correction necessary, I am not sure, but you will never obtain 30\% like for fainter meteoroids.

ARNOLD: I wish to remind the group that carbonaceous chondrites are by defini- 


\section{CEPLECHA}

tion objects which reach the earth. In this respect at least they differ from your groups $A$ or II. We must all help each other to avoid too close identification of either meteors or asteroidal surfaces or tiny particles collected in the upper atmosphere with our very detailed and specific information available to us from study of the meteorites in our collections.

CEPLECHA: I agree with this remark The A-group and the group II contain the majority of all observed meteoroids in the mass-range I spoke about. The average density of these bodies is less than that of the group $I$, the group which contains Pribram and Lost City meteorites, the only meteorite falls photographed. Also the structure of bodies of the group II is looser than that of the group $I$. On the other hand, the density is higher than that of the cocometary material, well identified from associations with parent comets. A distinct evidence of the presence of carbon in meteoroids of group II is spectroscopic. Thus $I$ should identify the A-group and the group II with hypothetical meteoroids outside the atmosphere having not so strong structure than ordinary chondrites and containing some carbon. The only similar material we know in the meteorite collections are carbonaceous chondrites CI. No doubt that the museum collections contain only a small portion of the carbonaceous meteoroid-population, if compared with the ordinary chondrites. And this small portion is the densest and the strongest.

WETHERILL: Possible identification with chondrites of $69 \%$ of the Prairie Network fireballs emphasizes a problem which has been around for a long time. This is that meteoroids or Apollo objects derived from the inner portion of the asteroid belt too frequently evolve into orbits which semi-major axis < $1.5 \mathrm{~A} . \mathrm{U}$., in disagreement with the fireball orbits. This suggests that these chondrites should be associated with more distant parts of the solar system, e.g., asteroids in the vicinity of the 2:1 Kirkwood gap, or cometary cores. These distant sources are, however, not popular from a cosmochemical or spectrophotometric point of view.

ANDERS: There is at least one strong argument against a cometary origin of carbonaceous chondrites. Many of them contain solar-wind gases and solar flare tracks, and since the flux of both decreases with the inverse square of the distance from the Sun, they cannot have formed at very great distances. In fact, from a comparison with similarly irradiated lunar soils, one can show that the distance cannot have been more than $3-4 \mathrm{AU}$. Moreover, $D$. Macdongall et al. have found that the irradiation took place prior to $4.2-4.3 \times 10^{9} \mathrm{yr}$, so the parent body must have been in or near the asteroid belt at that early time. 